\title{
Spectral signature barcodes based on S-shaped Split Ring Resonators (S-SRRs)
}

\author{
Cristian Herrojo*, Jordi Naqui, Ferran Paredes, and Ferran Martín \\ CIMITEC, Departament d'Enginyeria Electrònica, Universitat Autònoma de Barcelona, 08193 Bellaterra, Spain
}

Received 26 February 2016 / Accepted 26 April 2016

\begin{abstract}
In this paper, it is shown that S-shaped split ring resonators (S-SRRs) are useful particles for the implementation of spectral signature (i.e., a class of radiofrequency) barcodes based on coplanar waveguide (CPW) transmission lines loaded with such resonant elements. By virtue of its S shape, these resonators are electrically small. Hence S-SRRs are of interest for the miniaturization of the barcodes, since multiple resonators, each tuned at a different frequency, are used for encoding purposes. In particular, a 10-bit barcode occupying $1 \mathrm{GHz}$ spectral bandwidth centered at $2.5 \mathrm{GHz}$, with dimensions of $9 \mathrm{~cm}^{2}$, is presented in this paper.
\end{abstract}

Key words: Split ring resonators (SRRs), Radiofrequency barcodes, Chipless RFID.

\section{Introduction}

Metamaterial transmission lines can be implemented by loading a host line with electrically small resonant elements, such as split ring resonators (SRRs) [1], or complementary split ring resonators (CSRRs) [2], among others. Many microwave devices based on these artificial lines have been designed on the basis of the further controllability, as compared to ordinary lines, of line impedance and dispersion [3], achieved by virtue of the loading elements (which provide more degrees of freedom for device design). The key aspect in these designs is thus impedance and dispersion engineering. However, in other applications, the resonance phenomenon is the key aspect. In this case, where the functionality is based on particle resonance (rather than on impedance and dispersion engineering), the lines are usually referred to as transmission lines with metamaterial loading [3]. These structures have been applied to the design of planar bandstop and notch filters $[4,5]$, multiband printed dipole and monopole antennas [6-8], commonmode suppressed differential lines and filters $[9,10]$, microwave sensors [11-18], and radiofrequency barcodes [19-21], among others.

In this work, radiofrequency barcodes implemented by means of S-shaped SRRs (S-SRRs), previously used for the implementation of metamaterials [22-24], are presented in a

\footnotetext{
*e-mail: cristian.herrojo@uab.cat
}

journal paper for the first time. S-SRRs are electrically smaller than electric-LC resonators (ELC) and even smaller than split ring resonators (SRRs) [15]. Thus, high miniaturization levels can be obtained by using S-SRRs, with direct impact on the achievable number of bits per $\mathrm{cm}^{2}$, superior in S-SRR-based barcodes.

S-SRRs have been successfully applied to the design of microwave filters [25] and sensors [26] as well. Coupled to CPW transmission lines, S-SRRs generate notches (transmission zeros) in the transmission coefficient at their resonance frequency. However, through a proper orientation, line to resonator coupling can be prevented, and hence the presence or absence of the transmission zero can be controlled, and this is used for encoding, as will be later shown.

\section{Topology and working principle}

The typical topology of this particle is depicted in Figure 1. This particle cannot be excited by means of an uniform timevarying magnetic field applied in the direction orthogonal to the plane of the particle, contrary to SRRs [1,27], but it can be driven by means of counter magnetic fields, as depicted in the figure, and similar to the excitation of electric LC (ELC) resonant particles [28]. According to this, it follows that by etching these S-SRRs on the back substrate side of a coplanar waveguide $(\mathrm{CPW})$, the fundamental resonance can be 


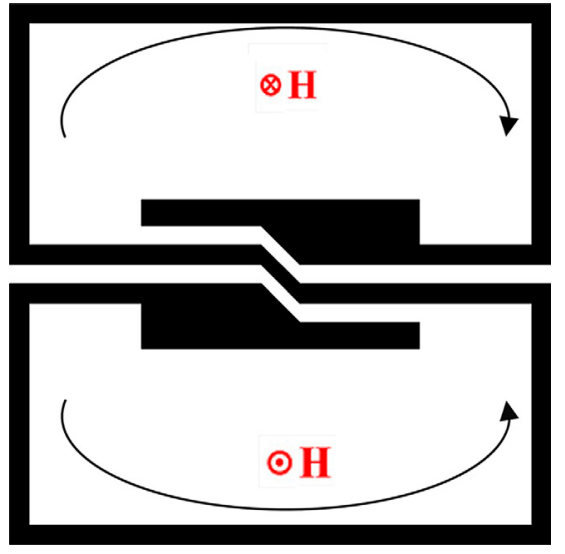

Figure 1. Typical topology of an S-SRR and indication of the driving mechanism by means of counter-magnetic fields.

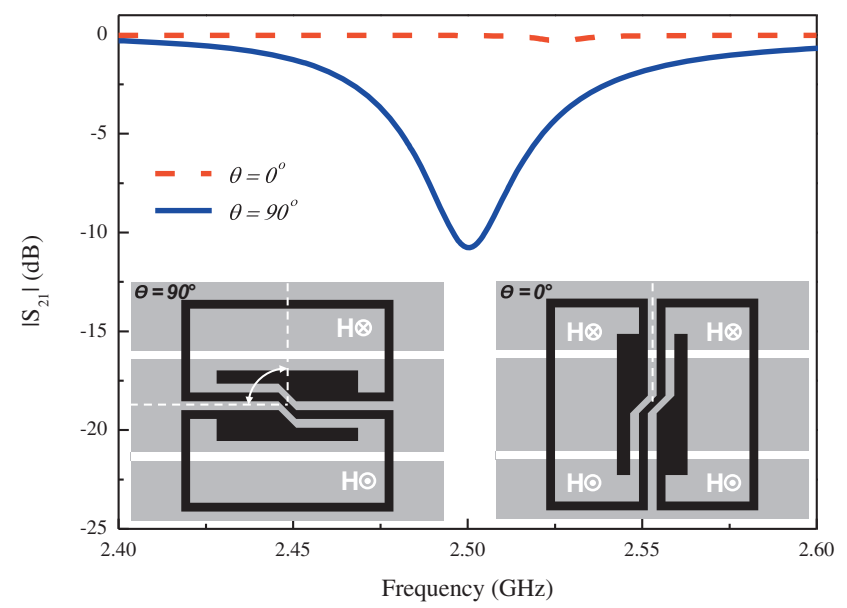

Figure 2. Response of the CPW loaded with a single S-SRR for the two considered states: " 1 " $\left(90^{\circ}\right)$ and " 0 " $\left(0^{\circ}\right)$.

excited as long as each loop of the particle lies on bottom of one of the slots of the CPW, as indicated in Figure 2. However, by rotating the particle $90^{\circ}\left(0^{\circ}\right.$ orientation in Figure 2), no significant excitation is expected since there is not a net magnetic field in each loop, able to induce a current. According to this, S-SRRs are useful for the implementation of multiresonator $[19,20]$ spectral signature barcodes for chipless RFID, where each resonator corresponds to a bit and the " 0 " or " 1 " logic states are simply set by the orientation of the S-SRRs.

\section{Circuit model and design}

The typical topology (including relevant dimensions) and circuit model of a CPW loaded with a square S-SRR, etched on the back substrate side, are depicted in Figure 3 [26]. The line is modeled by the inductance $L$ and the capacitance $C$, the S-SRR is described by the capacitance $C_{s}$ and by the inductance of each loop, $L_{s}$. Finally, the coupling between the line and the resonator is accounted for by the mutual inductance $2 M$. Note that this model is formally identical to the one describing a SRR-loaded CPW transmission line [1, 3]. As discussed in [26], the mutual coupling depends on the relative orientation between the line and the resonator, it being maximum for the $90^{\circ}$ orientation, i.e., the one indicated in Figure 3a. In the circuit of Figure 3b, losses have been excluded. This circuit can be transformed to the one indicated in Figure 3c, where the reactive elements of both models are related by [3]:

$$
\begin{gathered}
L_{s}^{\prime}=\frac{M^{2}}{2 L_{s}} \\
C_{s}^{\prime}=\frac{4 L_{s}^{2} C_{s}}{M^{2}} \\
L^{\prime}=L-L_{s}^{\prime}
\end{gathered}
$$

and the notch (angular) frequency is given by

$$
\omega_{0}=\frac{1}{\sqrt{2 L_{s} C_{s}}}=\frac{1}{\sqrt{L_{s}^{\prime} C_{s}^{\prime}}}
$$

This model has been validated by parameter extraction, following the procedure reported in [29]. Figure 4 shows the frequency response of a particular S-SRR-loaded CPW, where both the lossless electromagnetic (inferred from

(a)
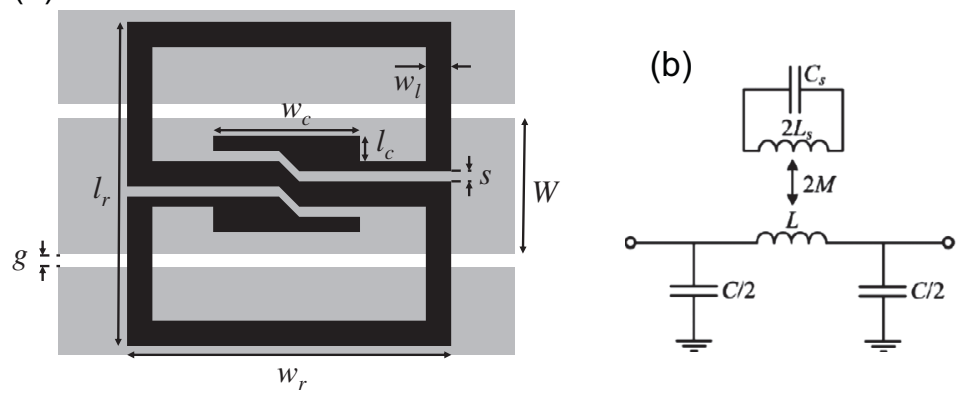

(c)

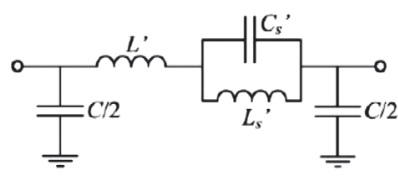

Figure 3. Topology of a S-SRR-loaded CPW (a), lumped element equivalent circuit model (b), and transformed model (c). 
(a)

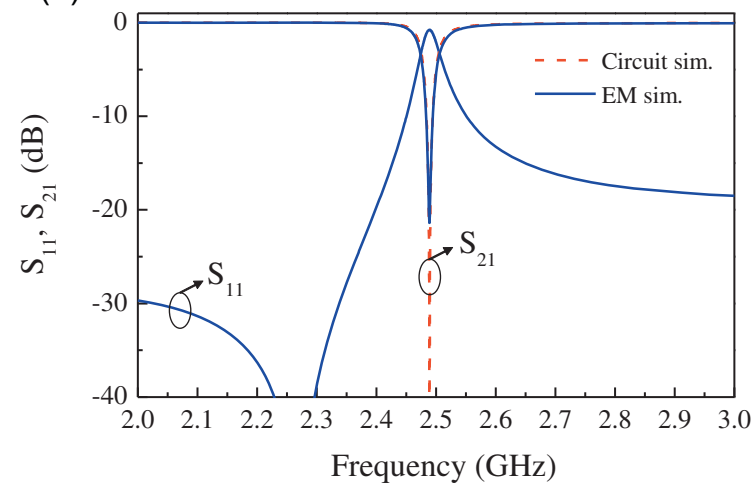

(b)

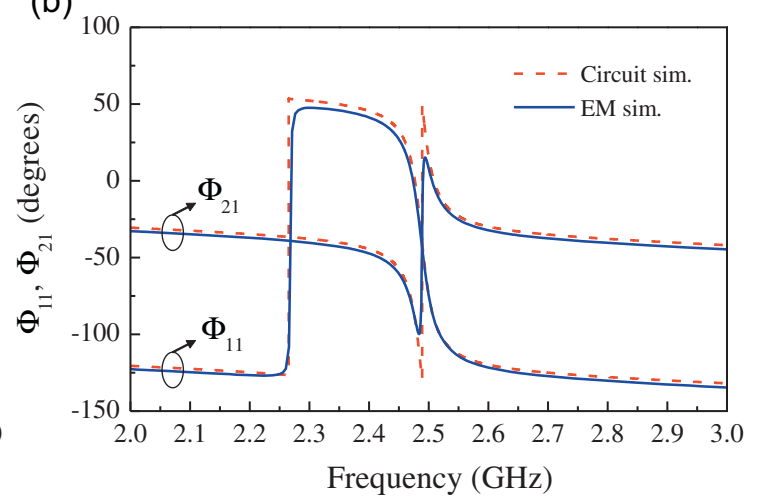

Figure 4. Frequency response of a CPW loaded with a square S-SRR. (a) Magnitude response; (b) phase response. Dimensions are: $l_{r}=w_{r}=5.6 \mathrm{~mm}, w_{l}=s=0.2 \mathrm{~mm}, w_{c}=2.87 \mathrm{~mm}$, and $l_{c}=0.47 \mathrm{~mm}$. Line dimensions are: $W=2.1 \mathrm{~mm}, g=0.2 \mathrm{~mm}$ corresponding to a $50 \Omega$ transmission line. The extracted element values are: $C=0.92 \mathrm{pF}, L^{\prime}=1.69 \mathrm{nH}, C_{s}{ }^{\prime}=58.6 \mathrm{pF}$, and $L_{s}{ }^{\prime}=69.8 \mathrm{pH}$.

(a)

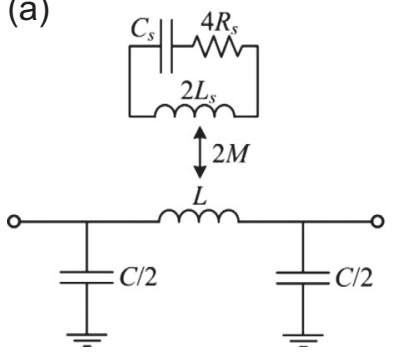

(b)

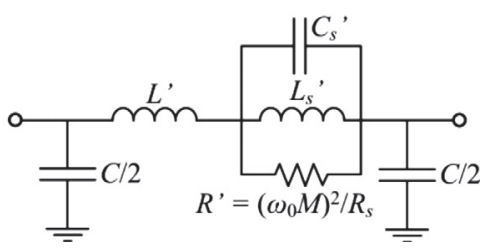

Figure 5. Lossy equivalent circuit model of an S-SRR-loaded CPW. (a) Simplified model and (b) equivalent transformed model.

Keysight Momentum) and circuit simulations are compared. The considered substrate is Rogers $R O 4003 C$ with dielectric constant $\varepsilon_{r}=3.55$ and thickness $h=0.81 \mathrm{~mm}$ (dimensions as well as circuit element values are indicated in the caption). As can be appreciated, there is very good agreement between the circuit and electromagnetic simulations in the frequency range of interest, pointing out the validity of the lumped element equivalent circuit model.

For design purposes, there are two important parameters: the notch depth, and the $-3 \mathrm{~dB}$ notch bandwidth. In order to accommodate the largest number of bits in a given spectral bandwidth, it is convenient to reduce the notch bandwidth of each individual resonator as much as possible, but, due to losses, this affects the notch depth, reducing its magnitude and consequently jeopardizing the correct reading of the written code. Therefore, a tradeoff results. To gain insight into this important aspect, let us now consider the presence of losses in the circuit models (Figure 5). The notch magnitude in the circuit of Figure 5b can be expressed as [26]:

$$
\left|S_{21}\right|(\mathrm{dB})=20 \log _{10}\left|\frac{1}{Z_{s}\left(\frac{Y_{p}^{2} Z_{0}}{2}+\frac{1}{2 Z_{0}}\right)+Y_{p}\left(Z_{s}+Z_{0}\right)+1}\right|
$$

where $Z_{s}$ is the impedance of the series branch, $Y_{p}$ is the admittance of the shunt branch, and $Z_{0}$ is the port impedance.
The previous expression, evaluated at resonance, can be written as:

$$
\begin{aligned}
& \left|S_{21}\right|(\mathrm{dB}) \\
& =20 \log _{10}\left|\frac{1}{\left(Z_{L^{\prime}}+\frac{\omega_{0}^{2} M^{2}}{R_{s}}\right)\left(\frac{Y_{p}^{2} Z_{0}}{2}+\frac{1}{2 Z_{0}}\right)+Y_{p}\left(Z_{L^{\prime}}+\frac{\omega_{0}^{2} M^{2}}{R_{s}}+Z_{0}\right)+1}\right|
\end{aligned}
$$

where $Z_{L^{\prime}}$ is the impedance of the line inductance. To the light of equation (6), $M$ tends to increase the notch depth, whereas an increase in $R_{s}$ reduces the magnitude of the notch, as expected. Nevertheless, $R_{s}$ is not a design parameter, contrary to $M$, which depends on S-SRR orientation and also on substrate thickness. Note that, according to expressions (1) and (2), if $M$ increases, $L_{s}{ }^{\prime}$ also increases, whereas $C_{s}{ }^{\prime}$ decreases, thereby enhancing the bandwidth (related to the ratio $\left.L_{s}^{\prime} / C_{s}^{\prime}\right)$.

By considering the substrate permittivity and thickness indicated before $\left(\varepsilon_{r}=3.55\right.$, and $\left.h=0.81 \mathrm{~mm}\right)$ and a loss tangent of $\tan \delta=0.0021$, we have obtained the frequency response of the S-SRR-loaded CPW of Figure 2, inferred from electromagnetic simulation with losses (the conductivity of copper for the metallic layers has been assumed). Then we have repeated these electromagnetic simulations by considering different substrate thicknesses, in order to infer the effect of $M$ on bandwidth and notch depth. The results are depicted 
(a)

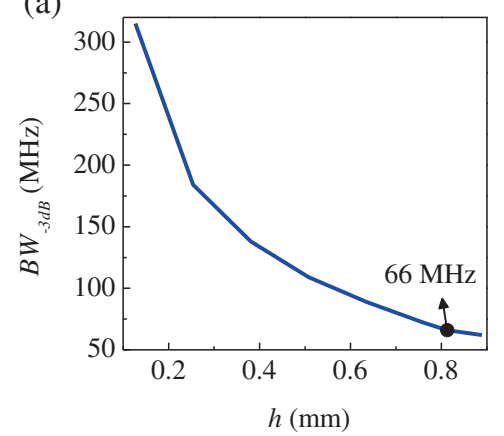

(b)

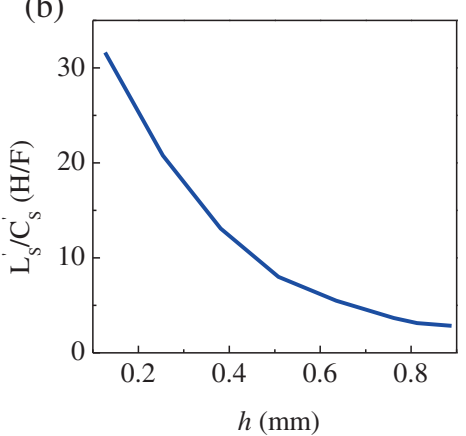

(c)

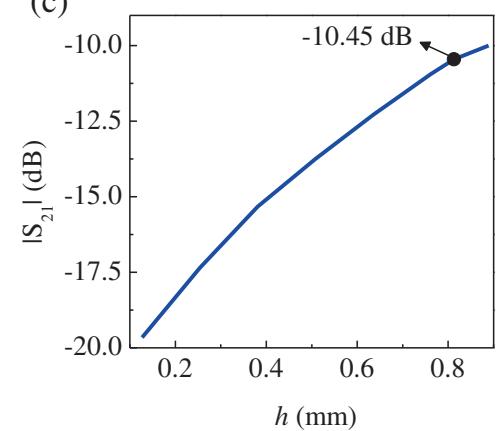

Figure 6. Dependence of the notch bandwidth (a), $L_{s}^{\prime} / C_{s}^{\prime}(\mathrm{b})$, and notch depth (c) with substrate thickness.
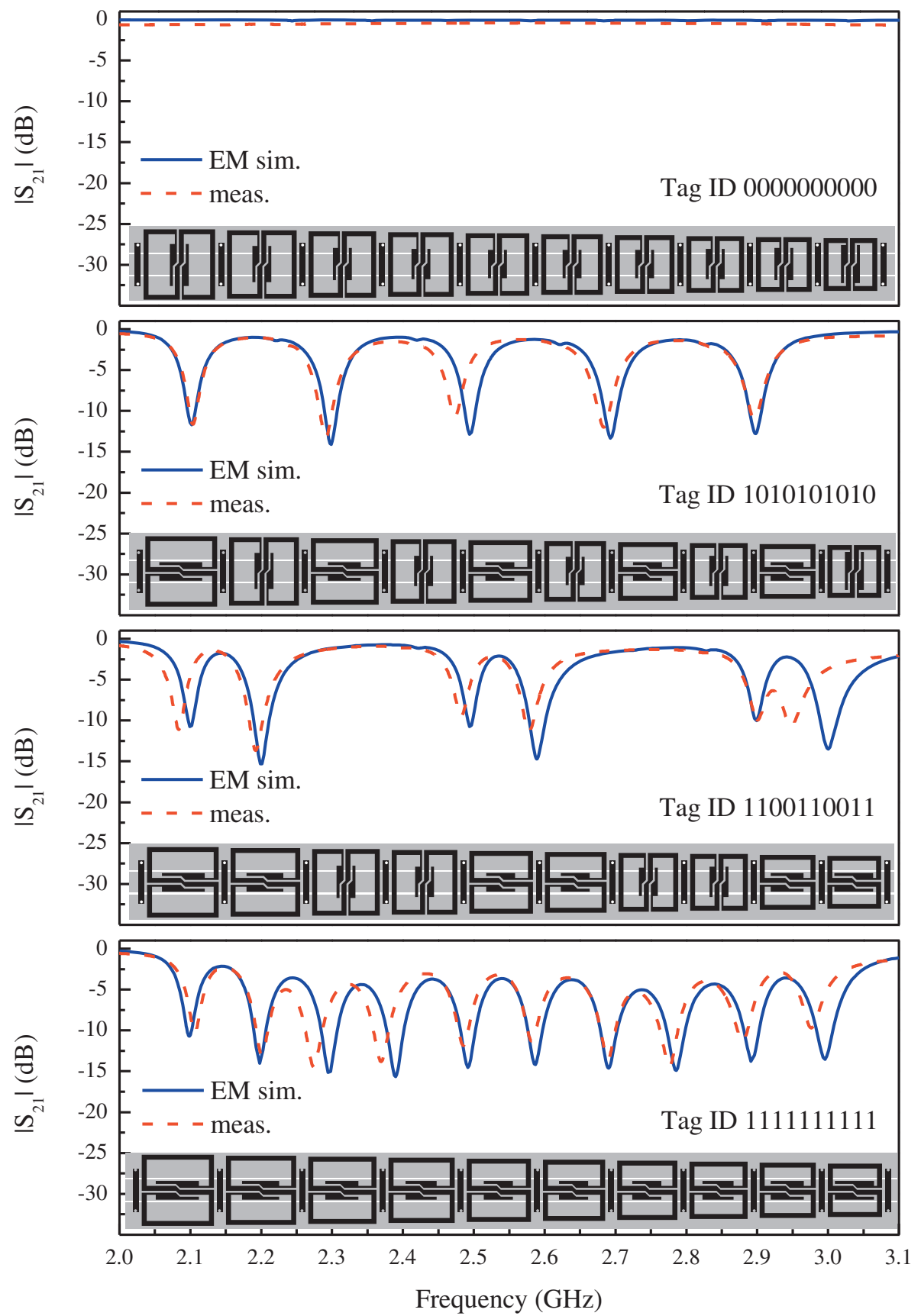

Figure 7. Simulated and measured transmission coefficient of the corresponding indicated codes. 
in Figure 6, where we have also included the dependence of $L_{s}{ }^{\prime} / C_{s}^{\prime}$ on substrate thickness. In order to accommodate 10 bits (and hence 10 resonators) in a $1 \mathrm{GHz}$ spectral bandwidth between $2 \mathrm{GHz}$ and $3 \mathrm{GHz}$, it is necessary to limit the resonator bandwidths to less than $100 \mathrm{MHz}$. In view of Figure 6, for the thickness of $h=0.81 \mathrm{~mm}$, the notch depth is found to be larger than $10 \mathrm{~dB}$, which is a reasonable value to adequately read the " 1 " logic states, whereas this thickness clearly gives a bandwidth much smaller than $100 \mathrm{MHz}$. Therefore, this has been the considered thickness value, which corresponds to a commercially available microwave substrate. From the considered resonator (with a frequency of $2.5 \mathrm{GHz}$ ), the other resonant elements have been obtained by merely scaling the length of the loops.

\section{Results}

The topology of the S-SRR with reference frequency has been determined with a relatively small value of $L_{s}$ and large value of $C_{s}$ (significant $L_{s}^{\prime} / C_{s}^{\prime}$ ratio) in order to achieve a notch depth of at least $10 \mathrm{~dB}$. The dimensions are $l_{r}=w_{r}=6.4 \mathrm{~mm}$, $w_{l}=0.5 \mathrm{~mm}, s=0.2 \mathrm{~mm}, w_{c}=2.9 \mathrm{~mm}$, and $l_{c}=0.5 \mathrm{~mm}$. After scaling, in order to generate notch frequencies equally distributed within the bandwidth $2-3 \mathrm{GHz}$, we have implemented several codes by loading the CPW with such resonant elements, where the " 1 " and " 0 ' logic states have been simply achieved by properly orienting the S-SRRs. The four considered codes and the corresponding frequency responses (inferred from lossy electromagnetic simulations and measurements) are depicted in Figure 7. Note that the ground plane regions of the CPW are connected by means of backside strips and vias in order to avoid the generation of the parasitic slot mode. The measured responses have been achieved by means of the Agilent N5221A vector network analyzer. According to Figure 7, there is good agreement between the electromagnetic simulation and measurement, and the different code responses reveal that a threshold of $5 \mathrm{~dB}$ suffices to distinguish between the " 0 " and " 1 " logic states. The area of the codes is as small as $9 \mathrm{~cm}^{2}$. Moreover, the fact that by rotating the S-SRR the coupling can be controlled opens the possibility of increasing the number of states per resonator, and thus increase the information density per $\mathrm{GHz}$ (work is in progress).

\section{Conclusions}

In summary, novel spectral signature barcodes implemented by loading a CPW with S-SRRs have been proposed. On the basis of the lumped element equivalent circuit model, which has been validated through parameter extraction, we have analyzed the effects of line to resonator coupling and losses on the response of the S-SRR loaded CPW. Particularly, it has been found that by increasing the mutual coupling, the notch magnitude of the structure is enhanced but at the expense of a broader bandwidth. Hence, it has been concluded that a tradeoff for design purposes is necessary, and with this in mind we have designed the S-SRRs in order to accommodate 10 notches (corresponding to 10 bits) in a $1 \mathrm{GHz}$ spectral bandwidth between $2 \mathrm{GHz}$ and $3 \mathrm{GHz}$. The resulting codes, smaller than $9 \mathrm{~cm}^{2}$, exhibit responses that can be easily read, with equidistant notches for the extreme case where all the bits are set to the "1" state.

Acknowledgements. This work was supported by MINECO-Spain (projects TEC2013-40600-R and RTC-2014-2550-7 ChiplessRFID), by Generalitat de Catalunya (project 2014SGR-157), by Institució Catalana de Recerca i Estudis Avançats (who awarded Ferran Martín), and by FEDER funds. Cristian Herrojo has been granted by MINECO with the grant number BES-2014-068164.

\section{References}

1. F. Martín, F. Falcone, J. Bonache, R. Marqués, M. Sorolla, Split ring resonator based left handed coplanar waveguide, Appl. Phys. Lett. 83 (2003) 4652-4654.

2. F. Falcone, T. Lopetegi, J.D. Baena, R. Marqués, F. Martín, M. Sorolla, Effective negative-epsilon stop-band microstrip lines based on complementary split ring resonators, IEEE Microw. Wirel. Compon. Lett. 14 (2004) 280-282.

3. F. Martín, Artificial Transmission Lines for RF and Microwave Applications, John Wiley, Hoboken, NJ, 2015.

4. F. Martín, F. Falcone, J. Bonache, R. Marqués, M. Sorolla, Miniaturized CPW stop band filters based on multiple tuned split ring resonators, IEEE Microw. Wirel. Compon. Lett. 13 (2003) 511-513.

5. J. García-García, J. Bonache, I. Gil, F. Martín, R. Marqués, F. Falcone, T. Lopetegi, M.A.G. Laso, M. Sorolla, Comparison of electromagnetic bandgap and split rings resonator microstrip lines as stop band structures, Microw. Opt. Technol. Lett. 44 (2005) 376-379.

6. F.J. Herraiz-Martínez, L.E. García-Muñoz, D. GonzálezOvejero, V. González-Posadas, D. Segovia-Vargas, Dualfrequency printed dipole loaded with split ring resonators, IEEE Antennas Wirel. Propag. Lett. 8 (2009) 137-140.

7. F.J. Herraiz-Martínez, G. Zamora, F. Paredes, F. Martín, J. Bonache, Multiband printed monopole antennas loaded with open complementary split ring resonators for PANs and WLANs, IEEE Antennas Wirel. Propag. Lett. 10 (2011) 1528-1531.

8. F.J. Herraiz-Martínez, F. Paredes, G. Zamora, F. Martín, J. Bonache, Dual-band printed dipole antenna loaded with open complementary split-ring resonators (OCSRRs) for wireless applications, Microw. Opt. Technol. Lett. 54 (2012) 1014-1017.

9. J. Naqui, A. Fernández-Prieto, M. Durán-Sindreu, F. Mesa, J. Martel, F. Medina, F. Martín, Common mode suppression in microstrip differential lines by means of complementary split ring resonators: theory and applications, IEEE Trans. Microwave Theor. Tech. 60 (2012) 3023-3034.

10. P. Vélez, J. Naqui, A. Fernández-Prieto, M. Durán-Sindreu, J. Bonache, J. Martel, F. Medina, F. Martín, Differential bandpass filter with common mode suppression based on open split ring resonators and open complementary split ring resonators, IEEE Microw. Wirel. Compon. Lett. 23 (1) (2013) 22-24.

11. M. Puentes, C. Weiss, M. Schüßler, R. Jakoby, Sensor array based on split ring resonators for analysis of organic tissues, IEEE MTT-S International Microwave Symposium Digest, Baltimore, MD, USA, 2011, pp. 5-10. 
12. J. Naqui, M. Durán-Sindreu, F. Martín, Novel sensors based on the symmetry properties of Split Ring Resonators (SRRs), Sensors 11 (2011) 7545-7553.

13. M.S. Boybay, O.M. Ramahi, Material characterization using complementary split-ring resonators, IEEE Trans. Instrum. Meas. 61 (2012) 3039-3046.

14. J. Naqui, M. Durán-Sindreu, F. Martín, Alignment and position sensors based on split ring resonators, Sensors 12 (2012) 11790-11797.

15. J. Naqui, F. Martín, Transmission lines loaded with bisymmetric resonators and their application to angular displacement and velocity sensors, IEEE Trans. Microwave Theor. Tech. 61 (2013) 4700-4713.

16. A. Abduljabar, D. Rowe, A. Porch, D. Barrow, Novel microwave microfluidic sensor using a microstrip split-ring resonator, IEEE Trans. Microwave Theor. Tech. 62 (2014) 679-688.

17. A. Ebrahimi, W. Withayachumnankul, S. Al-Sarawi, D. Abbott, High-sensitivity metamaterial-inspired sensor for microfluidic dielectric characterization, IEEE Sens. J. 14 (2014) 1345-1351.

18. A. Ebrahimi, W. Withayachumnankul, S.F. Al-Sarawi, D. Abbott, Metamaterial-inspired rotation sensor with wide dynamic range, IEEE Sens. J. 14 (2014) 2609-2614.

19. S. Preradovic, N.C. Karmakar, Chipless RFID: bar code of the future, IEEE Microw. Mag. 11 (2010) 87-97.

20. S. Preradovic, I. Balbin, N.C. Karmakar, G.F. Swiegers, Multiresonator-based chipless RFID system for low-cost item tracking, IEEE Trans. Microwave Theor. Tech. 57 (2009) 1411-1419.

21. J. Naqui, M. Durán-Sindreu, F. Martín, On the symmetry properties of coplanar waveguides loaded with symmetric resonators: analysis and potential applications, IEEE MTT-S Int. Microwave Symp. Dig, Montreal (Canada), 2012.

22. H. Chen, L. Ran, J. Huangfu, X. Zhang, K. Chen, T.M. Grzegorczyk, J.A. Kong, Left-handed materials composed of only S-shaped resonators, Phys. Rev. E 70 (2004) 057605.

23. H. Chen, L. Ran, J. Huangfu, X. Zhang, K. Chen, T.M. Grzegorczyk, J.A. Kong, Negative refraction of a combined double S-shaped metamaterial, Appl. Phys. Lett. 86 (2005) 151909.

24. H. Chen, L.X. Ran, J.T. Huang-Fu, X.-M. Zhang, K.-S. Cheng, T.M. Grzegorczyk, J.A. Kong, Magnetic properties of S-shaped split ring resonators, Progress Electromagn. Res. 51 (2005) 231-247.

25. A.K. Horestani, M. Durán-Sindreu, J. Naqui, C. Fumeaux, F. Martín, Compact coplanar waveguide band-pass filter based on coupled S-shaped split ring resonators, Microw. Opt. Technol. Lett. 57 (2015) 1113-1116.

26. J. Naqui, J. Coromina, A. Karami-Horestani, C. Fumeaux, F. Martín, Angular displacement and velocity sensors based on coplanar waveguides (CPWs) loaded with S-shaped split ring resonator (S-SRR), Sensors 15 (2015) 9628-9650.

27. J.B. Pendry, A.J. Holden, D.J. Robbins, W.J. Stewart, Magnetism from conductors and enhanced nonlinear phenomena, IEEE Trans. Microwave Theor. Tech. 47 (1999) 2075-2084.

28. D. Schurig, J.J. Mock, D.R. Smith, Electric-field-coupled resonators for negative permittivity metamaterials, Appl. Phys. Lett. 88 (2006) 041109.

29. F. Aznar, M. Gil, J. Bonache, J.D. Baena, L. Jelinek, R. Marqués, F. Martín, Characterization of miniaturized metamaterial resonators coupled to planar transmission lines, J. Appl. Phys. 104 (2008) 114501-1-8. 